\title{
Article
}

\section{Analysis of Fluorescence Decay Kinetics of Indocyanine Green Monomers and Aggregates in Brain Tumor Model In Vivo}

\author{
Dina Farrakhova ${ }^{1, *(1)}$, Igor Romanishkin ${ }^{1}\left(\mathbb{D}\right.$, Yuliya Maklygina ${ }^{1}$, Lina Bezdetnaya ${ }^{2,3}$ and Victor Loschenov ${ }^{1,4}$ \\ 1 Prokhorov General Physics Institute of the Russian Academy of Science, 119991 Moscow, Russia; \\ igor.romanishkin@nsc.gpi.ru (I.R.); us.samsonova@physics.msu.ru (Y.M.); loschenov@nsc.gpi.ru (V.L.) \\ 2 Centre de Recherche en Automatique de Nancy, CNRS, Université de Lorraine, 54519 Vandoeuvre-lès-Nancy, \\ France; 1.bolotine@nancy.unicancer.fr \\ 3 Institut de Cancérologie de Lorraine, 54519 Vandoeuvre-lès-Nancy, France \\ 4 Institute of Engineering Physics for Biomedicine, National Research Nuclear University MEPhI, \\ 115409 Moscow, Russia \\ * Correspondence: farrakhova.dina@mail.ru; Tel.: +7-968-587-52-75
}

\section{check for} updates

Citation: Farrakhova, D.; Romanishkin, I.; Maklygina, Y.; Bezdetnaya, L.; Loschenov, V. Analysis of Fluorescence Decay Kinetics of Indocyanine Green Monomers and Aggregates in Brain Tumor Model In Vivo. Nanomaterials 2021, 11, 3185. https://doi.org/ 10.3390/nano11123185

Academic Editors: Fiore P. Nicoletta and Francesca Iemma

Received: 30 October 2021

Accepted: 23 November 2021

Published: 24 November 2021

Publisher's Note: MDPI stays neutral with regard to jurisdictional claims in published maps and institutional affiliations.

Copyright: (c) 2021 by the authors. Licensee MDPI, Basel, Switzerland. This article is an open access article distributed under the terms and conditions of the Creative Commons Attribution (CC BY) license (https:/ / creativecommons.org/licenses/by/ $4.0 /)$.

\begin{abstract}
Spectroscopic approach with fluorescence time resolution allows one to determine the state of a brain tumor and its microenvironment via changes in the fluorescent dye's fluorescence lifetime. Indocyanine green (ICG) is an acknowledged infra-red fluorescent dye that self-assembles into stable aggregate forms (ICG NPs). ICG NPs aggregates have a tendency to accumulate in the tumor with a maximum accumulation at $24 \mathrm{~h}$ after systemic administration, enabling extended intraoperative diagnostic. Fluorescence lifetime analysis of ICG and ICG NPs demonstrates different values for ICG monomers and H-aggregates, indicating promising suitability for fluorescent diagnostics of brain tumors due to their affinity to tumor cells and stability in biological tissue.
\end{abstract}

Keywords: fluorescent diagnosis; fluorescent lifetime; near-infrared range; fluorescent dyes; H-aggregates; indocyanine green; brain cancer; glioma C6

\section{Introduction}

Any surgical intervention in the central nervous system for tumor resection requires high accuracy and selectivity of its effect on tissue. Currently, the main problem is the lack of a rapid, objective, and comprehensive intraoperative assessment of the boundaries of tumor tissue during surgical resection or a laser-induced therapy session [1]. The laser spectroscopic methods provide a unique opportunity to determine noninvasively the most significant parameters characterizing the condition of tissues and the prognosis of its possible evolutionary pathological changes, which may occur in the absence of timely treatment. Optical spectroscopy allows one to obain a wide range of information about physiological and morphological parameters $[2,3]$. This approach identifies a relationship between data from pathological tissue such as absorption, fluorescence, scattering caused by substances originally inherent to tissues and cells, and externally introduced markers. Laser spectroscopic methods with time resolution allow one to determine the state of a tumor and its microenvironment based on the changes in its photosensitizer (PS) fluorescence lifetime. The difference between the fluorescence lifetime is due to photosensitizer accumulation in cells with different phenotypes [4]. Immunocompetent cells, including macrophages, are a predominant component of brain tumors bioenvironment and allow an enhanced uptake of the photosensitizer molecules compared with cancer cells [5]. The change of fluorescence lifetime of ICG in cancer cells is due to a modification in the refractive index of biological tissues [6]. This approach has the advantage of being less sensitive to changes in the irradiation intensity and the dye concentration than the (relative) fluorescence intensities [7-9]. Noninvasive conditions for assessment of brain tumor tissue and surrounding tissues is important for performing a relapse-free operation without reducing the patient's quality of 
life. It is important to register small and fast functional changes to assess the relationship between surface metabolic and structural changes that occur during the formation and growth of brain cancer [10].

Exogenous fluorophores with selective accumulation in tumor cells and successive photo-cytotoxic effects can significantly increase the delineation of cancer boundaries and enhance their therapeutic effect. Indocyanine green (ICG) is a well-known infra-red fluorescent dye that is used extensively for vascular system imaging [11], tumor angiogenesis [11,12], and internal organs [13] imaging in vivo. ICG has an absorption in the far-red and near-infrared ranges, where biological components such as water and hemoglobin have low coefficients of absorption and scattering. ICG monomers and $\mathrm{H}$ - and J-aggregates have absorption peaks at $780 \mathrm{~nm}, 715 \mathrm{~nm}$, and $895 \mathrm{~nm}$, respectively, where penetration depth fluctuates from $0.5 \mathrm{~cm}$ to $0.8 \mathrm{~cm}$. Unfortunately, ICG is not preferable for tumor diagnosis due to limited accumulation and a high photobleaching effect [14]. There is a lot of research related to ICG embedded in different delivery system such as liposomes [15], polymers [16], lipid nanoparticles [17,18], albumin nanoparticles [19,20], and nanofibers [21], which significantly improve limited accumulation in vivo and photostability. High temperatures enable ICG to form stable $\mathrm{H}$ - and J-aggregates [22,23]. At present, there are numerous studies that use ICG colloidal solutions (ICG NPs) for cancer treatment [24-27]. ICG aggregates have a tendency to accumulate in the tumor, enabling extended intraoperative diagnostic. Moreover, after internalization of the aggregates by cells, it could be disassociated into the monomeric form of ICG [28], which can lead to the destruction of tumor tissue via photodynamic action.

\section{Materials and Methods}

Colloidal solution of ICG NPs and molecular solution of ICG were used as fluorescent dyes. ICG molecular form was heated at $65^{\circ} \mathrm{C}$ for $20 \mathrm{~h}$ to form ICG NPs according to a previously published method [28]. After forming ICG NPs, the solution was filtered through $0.40 \mu \mathrm{m}$ syringe filters to remove large aggregates. BALB/c mice with glioma C6 xenograft were used as experimental models. The experimental group consisted of 18 female mice with $20-23$ g body weight in the age range of $6-8$ weeks. The mice were kept in individually ventilated cages with controlled environmental values.

Animal care guidelines were used under the protocol of European Convention for the Protection of Vertebrate Animals used for Experimental and Other Scientific Purposes (Strasbourg, 18.III.1986). For spectroscopic research, the mice were restrained in tube restrainer configurations. Animals' supervision was conducted daily and efforts were made to prevent mice from suffering.

Fluorescent dyes of ICG and ICG NPs were administered intravenously into the tail vein at $10 \mathrm{mg} / \mathrm{kg}$ dose in $0.2 \mu \mathrm{L}$ volume for $30 \mathrm{~s}$. The concentrations of ICG and ICG NPs were selected corresponding to the doses used in clinical practice.

The fluorescence spectra of ICG and ICG NPs accumulated in tumor xenograft at different time intervals after fluorescent dyes administration were obtained via LESA-01Biospec spectroscopic system (Moscow, Russia). The excitation wavelength was $633 \mathrm{~nm}$ with $5 \mathrm{~mW} / \mathrm{cm}^{2}$ power density and a corresponding longpass filter was set at $635 \mathrm{~nm}$ for fluorescence registration. The filter used is convenient for fluorescence registration of ICG monomers and H-aggregates. A diffusely reflected signal for fluorescence curves was detected by optical fiber in contact of the distal tip to the tumor area and nearby healthy normal tissue (norma). At least three spectra for each measurement were obtained for statistical analysis. Excretory organs study was carried out after sacrificing the mice according to the protocol.

Fluorescence kinetics of ICG and ICG NPs were recorded by a system based on a Hamamatsu C10627-13 streak-camera (Iwata City, Japan) with a time resolution of 15 ps and laser with $637 \mathrm{~nm}$ excitation wavelength and $65 \mathrm{ps}$ pulse. The system works on a time-correlated single photon counting approach. The description of the system is reported in the [29]. The fiber was fixed on a tripod to maintain the working distance between 
the distal end and the tumor tissue. A $637 \mathrm{~nm}$ laser is preferable for excitation of ICG $\mathrm{H}$-aggregates, since it falls on the left shoulder of the absorption peak with the maximum at $715 \mathrm{~nm}$. The absorption peaks corresponding to ICG monomers and $\mathrm{H}$ - and J-aggregates are presented in our recent paper [30].

Statistical analysis was conducted with the paired Student's $t$-test for demonstration of significance of ICG monomers and H-aggregates, accumulated in tumor tissue. The empirical value is $\mathrm{t}=4.7$, greater than the critical one $\left(\mathrm{t}_{0.01}=2.78, \mathrm{t}_{0.05}=4.6\right)$.

\section{Results}

The accumulation kinetics of ICG and ICG NPs accumulation in tumor xenografts was assessed according to the fluorescence intensity. Fluorescence spectra were also obtained in the skin, which served as normal tissue sample (Figure 1a). The distribution of the fluorescent dye in excretory organs was obtained at times corresponding to the maximum of its accumulation (Figure 1b,c).

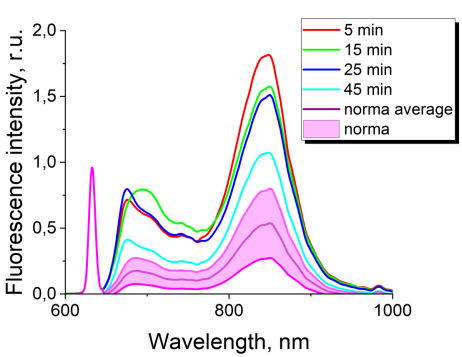

(a)

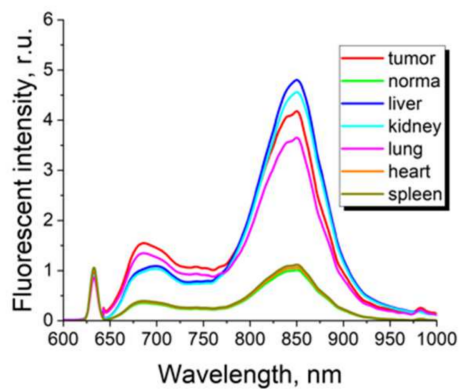

(b)

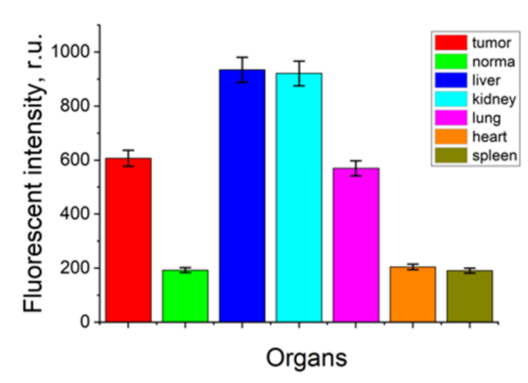

(c)

Figure 1. (a) Fluorescence spectra of ICG molecular solution in tumor xenografts at different times $\left(\lambda_{\mathrm{ex}}=633 \mathrm{~nm}\right)$. The data obtained from the study of normal skin samples at all time points fluctuate in the range between two spectral curves (pink color); (b) fluorescence spectra of ICG molecular solution in excretory organs 5 min after intravenous administration $\left(\lambda_{\mathrm{ex}}=633 \mathrm{~nm}\right)$; (c) integral dependence of the spectral curve of ICG molecular solution in tumor xenografts 5 min after intravenous administration $\left(\lambda_{\mathrm{ex}}=633 \mathrm{~nm}\right)$.

The maximum accumulation of ICG in tumor tissue was observed within the first $5 \mathrm{~min}$ after intravenous injection and then the fluorescent dye was rapidly eliminated (Figure 1a). To assess the accumulation of ICG in the excretory organs at the time corresponding to ICG maximum accumulation in the tumor, we registered the fluorescence spectra and the distribution of the fluorescence intensity in the organs and in the skin (taken as normal tissue) (Figure $1 b, c)$. Thus, the absence of significant contrast of ICG is demonstrated since the level of accumulation in the tumor slightly exceeds the level of total accumulation in the skin, while the level of ICG accumulation in the liver and kidneys is the highest, which confirms the presence of rapid elimination processes. The obtained results are consistent with other studies where ICG is used in the clinic as a contrast agent for visualization of the vascular network, which is permissible due to its rapid elimination [11-13].

The spectral analysis showed that ICG NPs accumulation in tumor tissue was maximal at $24 \mathrm{~h}$ after systemic administration (Figure 2a). Only H-aggregates of ICG were observed at $633 \mathrm{~nm}$ excitation after ICG NPs administration corresponding to the fluorescent maximum at $705 \mathrm{~nm}$. The obtained dynamics allow one to conclude that the aggregates enable the fluorescent dye to accumulate and stay in the tumor for a long time (up to $24 \mathrm{~h}$ post-administration). It should be noted that the comparison analysis of the fluorescence of ICG and ICG NP at the same time points was not possible. The molecular form of ICG has the rapid clearance by the liver within $1 \mathrm{~h}$ after intravenous administration with a rapid successive decline, while the ICG NP fluorescent signal peaks at $5 \mathrm{~h}$ after intravenous administration. There is an obvious mismatch between peaks of ICG molecular form and ICG NPs accumulation. The excretory organs analysis demonstrated that H-type aggregates of ICG NP noticeably accumulate in tumor tissue compared with ICG molecular 
form (Figure $2 b, c)$. As a result of the study, it is worth emphasizing that the aggregated form of ICG NPs is promising from the point of view of its spectral-fluorescent properties, as well as the local contrast accumulation of the nanoform in the tumor.

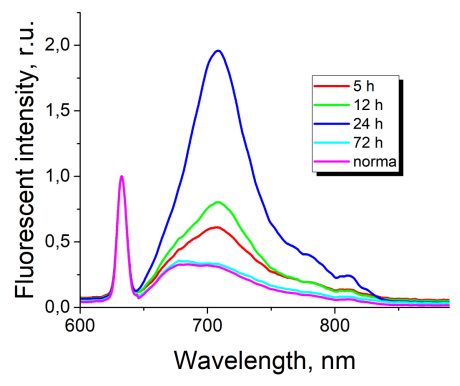

(a)

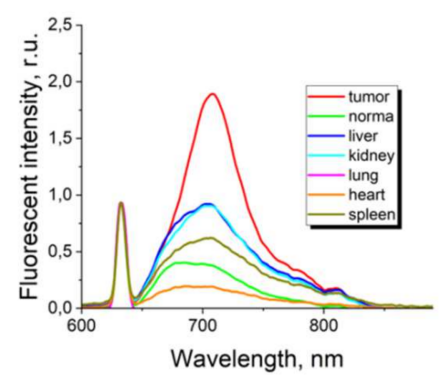

(b)

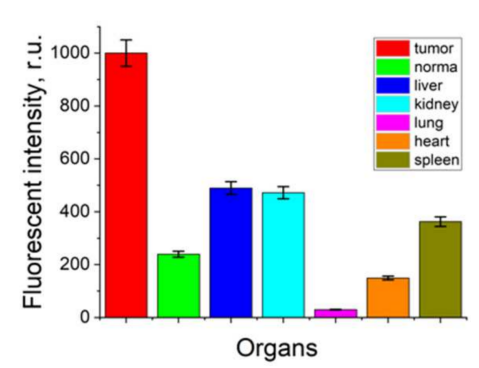

(c)

Figure 2. (a) Fluorescence spectra of ICG NPs colloidal solution in tumor xenografts at different times $\left(\lambda_{\mathrm{ex}}=633 \mathrm{~nm}\right)$; (b) fluorescence spectra of ICG NPs colloidal solution in excretory organs $24 \mathrm{~h}$ after intravenous administration $\left(\lambda_{\mathrm{ex}}=633 \mathrm{~nm}\right)$; (c) integral dependence of the spectral curve of ICG NPs colloidal solution in tumor xenografts under $24 \mathrm{~h}$ after intravenous administration $\left(\lambda_{\mathrm{ex}}=633 \mathrm{~nm}\right)$.

The fluorescence lifetimes of ICG and ICG NPs were obtained via the streak camera at times matching the maximal dyes' accumulation in the tumor (Figure 3).

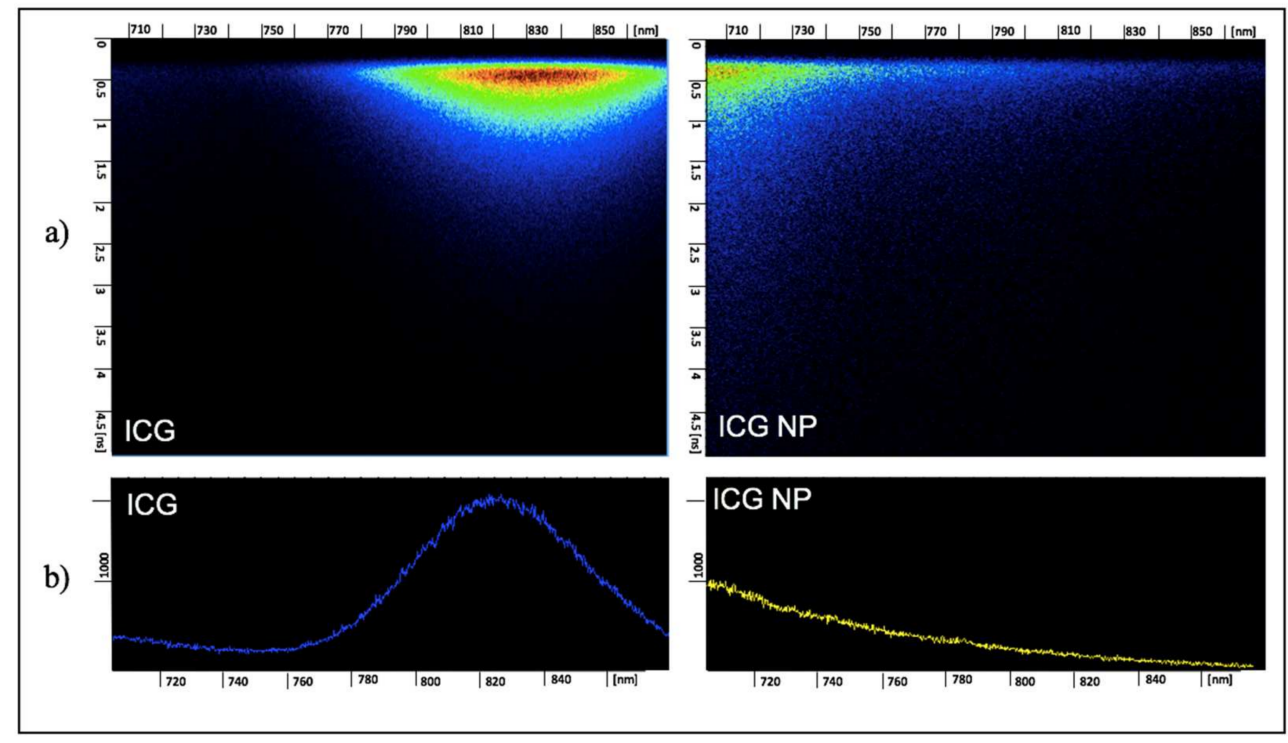

Figure 3. (a) Fluorescence spectra of fluorescence of ICG and ICG NPs accumulated in tumor xenograft; (b) profiles of fluorescence spectra of ICG and ICG NPs accumulated in tumor xenograft.

The spectra of fluorescence lifetime illustrate a fluorescence shoulder of ICG at 700-730 nm, which corresponds to H-aggregates, and an intense fluorescent peak of ICG at 790-860 nm, which corresponds to monomers. For ICG NPs, the fluorescence signal was registered at 700-730 $\mathrm{nm}$, which corresponded to ICG H-aggregates.

The fitting of the obtained fluorescence decay kinetics after the laser pulse excitation was approximated by an exponential function:

$$
I(t)=A_{1} e^{-\frac{x}{t 1}}+A_{2} e^{-\frac{x}{t 2}}+\cdots
$$

where $A_{1}, A_{2}, \ldots$ are the amplitude components of the fluorescent lifetime, directly proportional to the contribution of each exponential component; $t_{1}, t_{2}, \ldots$ are the corresponding fluorescent lifetime indicators, measured in nanoseconds. 
The fluorescence lifetimes of ICG accumulated in tumor xenografts were obtained for $\mathrm{H}$-aggregates and monomers separately, while ICG NPs fluorescence lifetimes were obtained for $\mathrm{H}$-aggregates. Mathematical fitting of fluorescence kinetic spectra demonstrated the availability of two fluorescence lifetimes for each form of ICG. For each sample five spectra were recorded, which were averaged to perform statistical analysis (Table 1). The second column of the Table presents two components of ICG fluorescence lifetimes (ns).

Table 1. Fluorescence lifetime of ICG in monomeric and aggregate forms in tumor and healthy tissue.

\begin{tabular}{ccc}
\hline Sample & Fluorescence Lifetime $\boldsymbol{\tau}, \mathbf{n s}$ & $\begin{array}{c}\text { Amplitude of } \\
\text { Fluorescence Lifetime, } \%\end{array}$ \\
\hline ICG monomers in tumor & $0.55 \pm 0.09$ & $21 \%$ \\
ICG monomers in norma & $0.82 \pm 0.03$ & $79 \%$ \\
ICG H-aggregates in tumor & $0.37 \pm 0.09$ & $64 \%$ \\
& $0.75 \pm 0.05$ & $36 \%$ \\
ICG H-aggregates in norma & $0.28 \pm 0.04$ & $83 \%$ \\
ICG NPs H-aggregates in tumor & $1.10 \pm 0.09$ & $17 \%$ \\
& $0.27 \pm 0.08$ & $82 \%$ \\
ICG NPs H-aggregates in norma & $1.21 \pm 0.11$ & $18 \%$ \\
& $0.26 \pm 0.03$ & $91 \%$ \\
\hline
\end{tabular}

The obtained data show the distinction between fluorescence lifetime values of ICG and different forms of ICG NPs accumulated in tumor tissue. The short component of the fluorescence lifetime of the monomeric form is significantly different from the short component of the H-aggregates. The statistical analysis shows statistically significant differences between the monomers and $\mathrm{H}$-aggregates in the molecular solution. Additionally, the long component of monomers is lower in comparison with the long component of $\mathrm{H}$-aggregates. The obtained data were compared with fluorescence lifetimes in normal tissue after intravenous administration. The fluorescence decay kinetics in tumor tissue are slightly different compared to intact tissue. The fluorescence lifetime of ICG monomeric form in the tumor for both components is slightly higher compared to normal tissue. Meanwhile, $\mathrm{H}$-aggregate values vary within the error margin in tumor and normal tissue.

\section{Discussion}

ICG has significant impact for intraoperative fluorescence navigation in the NearInfrared-II "window" (1000-1700 nm), especially in brain malignances [10,31,32]. However it has drawbacks like elimination from circulation and low photostability. In addition, once in circulation ICG is rapidly captured by liver, thus limiting its delivery to other sides. This is why the colloidal solution of ICG should be considered due to its stability. The results of the dynamics of the fluorescence signal under $633 \mathrm{~nm}$ laser excitation in the tumor after intravenous administration of ICG molecular form demonstrated the maximum fluorescence signal immediately after administration ( $0-5 \mathrm{~min})$, followed by its uniform elimination within an hour. The studied fluorescent dye ICG in molecular form has promising spectral-fluorescent characteristics necessary for the excitation of deep layers of biological tissues. The fluorescence spectra of ICG NPs in xenografted mice at different time intervals after systemic administration were recorded using $\lambda=633 \mathrm{~nm}$, which effectively excite $\mathrm{H}$-aggregates for visualization. We demonstrated the kinetics of accumulation of the fluorescent dye ICG NPs, and at the same time, the processes of its interaction with the biological tissue were controlled. The development and testing of ICG NPs colloidal solution is promising in order to increase its specificity to the tumor. ICG molecular form and ICG NPs have various accumulation times in tumor tissue. The fluorescence of ICG molecular form is observed only in the liver within one hour after intravenous administration, while ICG NPs fluorescent signal is detectable in the tumor from $5 \mathrm{~h}$ 
after intravenous administration. The presence of the ICG aggregates is not detectable immediately, but on average, $24 \mathrm{~h}$ after intravenous administration demonstrating an intense fluorescent signal in tumor tissue. ICG NPs are expected to be preferentially accumulated by the enhanced permeability and retention effect (EPR) similar to proteinbound ICG molecular form [33]. We hypothesize that aggregates are not internalized by tumor cells but are retained in the extracellular matrix due to surface negative charge of aggregated forms [28]. It was found that ICG NPs tend to be eliminated from the body by the reticuloendothelial system. As we showed in our recent study [30], a high concentration of ICG colloidal solution is observed in the liver and kidneys, indicating the metabolic pathway.

Fluorescence lifetime analysis of ICG and ICG NPs demonstrate different lifetime components for ICG monomers and H-aggregates. The first fluorescence lifetime component of ICG H-aggregate is twice as short as that of the monomer, while the second component of H-type aggregate is longer compared to monomers. According to these data, fluorescent decay kinetics allow to get information on tumor tissue and its bioenvironment. ICG H-aggregates selectively accumulate in tumor tissue, resulting in a clear advantage for long-lasting fluorescent diagnosis. The monomeric form of ICG is mostly used as a contrast agent for visualization of the vascular network, enabling predicting of metastasis ways. While ICG nanoparticles are very promising for fluorescent diagnostics of brain tumors due to their spectroscopic properties and a high selectivity, thus sparing healthy brain tissue.

\section{Conclusions}

To conduct a comparative analysis of ICG in molecular and colloidal solutions, the interstitial distribution of the fluorescent dye was studied in pre-clinical models. The accumulation maximum for ICG and ICG NPs were established in the tumor tissue, in normal tissue, and in the excretory organs. The obtained results showed different accumulation times of the studied fluorescent dyes in the pathological tissue: the molecular form of ICG accumulates within $5 \mathrm{~min}$, while ICG NPs accumulation takes $24 \mathrm{~h}$. It was demonstrated that ICG NPs are promising for fluorescence diagnostics due to their unique optical properties and selective accumulation. The analysis of fluorescence lifetime illustrates the difference between lifetime components allowing to separate ICG monomers and $\mathrm{H}$-aggregates in biological tissue. ICG colloidal solutions have significant prospects for fluorescent diagnostics of brain tumors due to their affinity to tumor cells and stability in biological tissue.

Author Contributions: Conceptualization, D.F. and Y.M.; methodology, V.L.; software, I.R.; validation, D.F. and I.R.; formal analysis, L.B.; investigation, V.L.; resources, D.F.; data curation, I.R.; writing—original draft preparation, Y.M.; writing—-review and editing, L.B.; visualization, D.F.; supervision, V.L.; project administration, V.L.; funding acquisition, Y.M. All authors have read and agreed to the published version of the manuscript.

Funding: The reported study was funded by RFBR according to the research project No. 21-52-15025 and partly by IEA (International Emerging Action) CNRS grant 00534.

Institutional Review Board Statement: Animal care guidelines were used under the protocol of European Convention for the Protection of Vertebrate Animals used for Experimental and Other Scientific Purposes (Strasbourg, 18.III.1986).

Informed Consent Statement: Not applicable.

Data Availability Statement: Not applicable.

Conflicts of Interest: The authors declare no conflict of interest. 


\section{References}

1. Nagaya, T.; Nakamura, Y.A.; Choyke, P.L.; Kobayashi, H. Fluorescence-guided surgery. Front. Oncol. 2017, 7, 314. [CrossRef] [PubMed]

2. Endo, H.; Owada, S.; Inagaki, Y.; Shida, Y.; Tatemichi, M. Metabolic reprogramming sustains cancer cell survival following extracellular matrix detachment. Redox Biol. 2020, 36, 101643. [CrossRef] [PubMed]

3. Muller, P.J.; Wilson, B.C. Photodynamic Therapy//Neurooncology: The Essentials; Thieme: New York, NY, USA, $2000 ;$ pp. $249-256$.

4. Ma, H.; Gao, Z.; Yu, P.; Shen, S.; Liu, Y.; Xu, B. A dual functional fluorescent probe for glioma imaging mediated by blood-brain barrier penetration and glioma cell targeting. Biochem. Biophys. Res. Commun. 2014, 449, 44-48. [CrossRef] [PubMed]

5. Korbelik, M.; Krosl, G. Photofrin accumulation in malignant and host cell populations of various tumours. Br. J. Cancer 1996, 73, 506-513. [CrossRef]

6. Suhling, K.; French, P.M.; Phillips, D. Time-resolved fluorescence microscopy. Photochem. Photobiol. Sci. 2005, 4, 13-22. [CrossRef]

7. Grabolle, M.; Kapusta, P.; Nann, T.; Shu, X.; Ziegler, J.; Resch-Genger, U. Fluorescence lifetime multiplexing with nanocrystals and organic labels. Anal. Chem. 2009, 81, 7807-7813. [CrossRef]

8. Raymond, S.B.; Boas, D.A.; Bacskai, B.J.; Kumar, A.T.N. Lifetime-based tomographic multiplexing. J. Biomed. Opt. $2010,15,046011$. [CrossRef] [PubMed]

9. Ehlert, O.; Thomann, R.; Darbandi, M.; Nann, T. A four-color colloidal multiplexing nanoparticle system. ACS Nano 2008, 2, 120-124. [CrossRef] [PubMed]

10. Cho, S.S.; Salinas, R.; Lee, J.Y.K. Indocyanine-Green for Fluorescence-Guided Surgery of Brain Tumors: Evidence, Techniques, and Practical Experience. Front. Surg. 2019, 6, 11. [CrossRef]

11. Zhang, H.F.; Maslov, K.; Stoica, G.; Wang, L.V. Functional Photoacoustic Microscopy for High-Resolution and Noninvasive In Vivo Imaging. Nat. Biotechnol. 2006, 24, 848-851. [CrossRef]

12. Diot, G.; Metz, S.; Noske, A.; Liapis, E.; Schroeder, B.; Ovsepian, S.V.; Meier, R.; Rummeny, E.; Ntziachristos, V. Multispectral Optoacoustic Tomography (MSOT) of Human Breast Cancer. Clin. Cancer Res. 2017, 23, 6912-6922. [CrossRef] [PubMed]

13. Song, K.H.; Wang, L.V. Deep Reflection-Mode Photoacoustic Imaging of Biological Tissue. J. Biomed. Opt. 2007, 12, 060503. [CrossRef] [PubMed]

14. Desmettre, T.; Devoisselle, J.; Mordon, S. Fluorescence Properties and Metabolic Features of Indocyanine Green (ICG) as Related to Angiography. Surv. Ophthalmol. 2000, 45, 15-27. [CrossRef]

15. Yan, F.; Wu, H.; Liu, H.; Deng, Z.; Liu, H.; Duan, W.; Liu, X.; Zheng, H. Molecular imaging-guided photothermal/photodynamic therapy against tumor by iRGD-modified indocyanine green nanoparticles. J. Control Release 2016, 224, 217-228. [CrossRef] [PubMed]

16. Ma, Y.; Tong, S.; Bao, G.; Gao, C.; Dai, Z. Indocyanine green loaded SPIO nanoparticles with phospholipid-PEG coating for dual-modal imaging and photothermal therapy. Biomaterials 2013, 34, 7706-7714. [CrossRef]

17. Zheng, M.; Yue, C.; Ma, Y.; Gong, P.; Zhao, P.; Zheng, C.; Sheng, Z.; Zhang, P.; Wang, Z.; Cai, L. Single-step assembly of DOX/ICG loaded lipid-polymer nanoparticles for highly effective chemo-photothermal combination therapy. ACS Nano 2013, 7, $2056-2067$. [CrossRef]

18. Navarro, F.P.; Berger, M.; Guillermet, S.; Josserand, V.; Guyon, L.; Neumann, E.; Vinet, F.; Texier, I. Lipid nanoparticle vectorization of indocyanine green improves fluorescence imaging for tumor diagnosis and lymph node resection. J. Biomed. Nanotechnol. 2012, 8, 730-741. [CrossRef] [PubMed]

19. Sheng, Z.; Hu, D.; Zheng, M.; Zhao, P.; Liu, H.; Gao, D.; Gong, P.; Gao, G.; Zhang, P.; Ma, Y.; et al. Smar human serum albumin-indocyanine green nanoparticles generated by programmed assembly for dual-modal imaging-guided cancer synergistic phototherapy. ACS Nano 2014, 8, 12310-12322. [CrossRef]

20. Chen, Q.; Liang, C.; Wang, X.; He, J.; Li, Y.; Liu, Z. An albumin-based theranostic nano-agent for dual-modal imaging guided photothermal therapy to inhibit lymphatic metastasis of cancer post surgery. Biomaterials 2014, 35, 9355-9362. [CrossRef] [PubMed]

21. Huang, P.; Gao, Y.; Lin, J.; Hu, H.; Liao, H.-S.; Yan, X.; Tang, Y.; Jin, A.; Song, J.; Niu, G.; et al. Tumor-specific formation of enzyme-instructed supramolecular self-assemblies as cancer theranostics. ACS Nano 2015, 9, 9517-9527. [CrossRef]

22. Belfield, K.D.; Bondar, M.V.; Hernandez, F.E.; Przhonska, O.V.; Yao, S. Two-photon absorption of a supramolecular pseudoisocyanine J-aggregate assembly. Chem. Phys. 2006, 320, 118-124. [CrossRef]

23. Yao, H.; Domoto, K.; Isohashi, T.; Kimura, K. In situ detection of birefringent mesoscopic H and J aggregates of thiacarbocyanine dye in solution. Langmuir 2005, 21, 1067-1073. [CrossRef] [PubMed]

24. Lovell, J.F.; Jin, C.S.; Huynh, E.; Jin, H.; Kim, C.; Rubinstein, J.L.; Chan, W.C.W.; Cao, W.; Wang, L.V.; Zheng, G. Porphysome nanovesicles generated by porphyrin bilayers for use as multimodal biophotonic contrast agents. Nat. Mater. 2011, 10, 324-332. [CrossRef] [PubMed]

25. Shakiba, M.; Ng, K.K.; Huynh, E.; Chan, H.; Charron, D.M.; Chen, J.; Muhanna, N.; Foster, F.S.; Wilsom, B.C.; Zheng, G. Stable J-aggregation enabled dual photoacoustic and fluorescence nanoparticles for intraoperative cancer imaging. Nanoscale 2016, 8 , 12618-12625. [CrossRef] [PubMed]

26. Song, X.; Gong, H.; Liu, T.; Cheng, L.; Wang, C.; Sun, X.; Liang, C.; Liu, Z. J-aggregates of organic dye molecules complexed with iron oxide nanoparticles for imaging-guided photothermal therapy under 915-nm light. Small 2014, 10, 4362-4370. [CrossRef] 
27. Song, X.; Zhang, R.; Liang, C.; Chen, Q.; Gong, H.; Liu, Z. Nano-assemblies of J-aggregates based on a NIR dye as a multifunctional drug carrier for combination cancer therapy. Biomaterials 2015, 57, 84-92. [CrossRef] [PubMed]

28. Liu, R.; Tang, J.; Xu, Y.; Zhou, Y.; Dai, Z. Nano-sized indocyanine green J-aggregate as a one-component theranostic agent. Nanotheranostics 2017, 1, 430. [CrossRef] [PubMed]

29. Bystrov, F.G.; Makarov, V.I.; Pominova, D.V.; Ryabova, A.V.; Loschenov, V.B. Analysis of photoluminescence decay kinetics of aluminum phthalocyanine nanoparticles interacting with immune cells. Biomed. Photonics 2016, 5, 3-8. [CrossRef]

30. Farrakhova, D.; Maklygina, Y.; Romanishkin, I.; Yakovlev, D.; Plyutinskaya, A.; Bezdetnaya, L.; Loschenov, V. Fluorescence imaging analysis of distribution of indocyanine green in molecular and nanoform in tumor model. Photodiagnosis Photodyn. Ther. 2021, in press. [CrossRef] [PubMed]

31. Zhu, S.; Tian, R.; Antaris, A.L.; Chen, X.; Dai, H. Near-infrared-II molecular dyes for cancer imaging and surgery. Adv. Mater. 2019, 31, 1900321. [CrossRef]

32. Teng, C.W.; Cho, S.S.; Singh, Y.; De Ravin, E.; Somers, K.; Buch, L.; Bream, S.; Singhal, S.; Delikatny, J.; Lee, J.Y. Second Window ICG Predicts Gross Total Resection and Progression Free Survival During Brain Metastasis Surgery. Neurosurgery 2020, 67, 1026-1035. [CrossRef] [PubMed]

33. Belykh, E.; Shaffer, K.V.; Lin, C.; Byvaltsev, V.A.; Preul, M.C.; Chen, L. Blood-brain barrier, blood-brain tumor barrier, and fluorescence-guided neurosurgical oncology: Delivering optical labels to brain tumors. Front. Oncol. 2020, 10, 739. [CrossRef] [PubMed] 